\title{
Adaptive Data Hiding in Compressed Video Domain
}

\author{
Arijit Sur and Jayanta Mukherjee \\ Department of Computer Science and Engineering, \\ Indian Institute of Technology, Kharagpur \\ \{arijits, jay\}@cse.iitkgp.ernet.in
}

\begin{abstract}
In this paper we propose a new adaptive block based compressed domain data hiding scheme which can embed relatively large number of secret bits without significant perceptual distortion in video domain. Macro blocks are selected for embedding on the basis of low inter frame velocity. From this subset, the blocks with high prediction error are selected for embedding. The embedding is done by modifying the quantized DCT AC coefficients in the compressed domain. The number of coefficients (both zero and non zero) used in embedding is adaptively determined using relative strength of the prediction error block. Experimental results show that this blind scheme can embed a relatively large number of bits without degrading significant video quality with respect to Human Visual System (HVS).
\end{abstract}

\section{Introduction}

With the enormous advancement of the multimedia technology such as High definition television (HDTV), Video on Demand (VOD), Video telephony etc, multimedia security becomes a significant issue nowadays. Video watermarking is one of possible countermeasure defending the world wide video piracy. Again secret communication through video stream is another interesting field in most modern era of information hiding. So data hiding in streaming media become an important research aspect today. There are number of video watermarking techniques are proposed in the recent literature. Three major trends can be classified such as extension of still image watermarking scheme, exploiting another temporal dimension such as motion and using standard video compression techniques such as MPEG standards. The extension of still image watermarking schemes are mostly based on spatial domain embedding which is computationally intensive and suffers from lack of robustness due to the inherent lossy nature of the video compression. According to the recent literature of compressed domain video watermarking, embedding is generally done either by modifying motion vector information or using prediction error. The motion vector based video watermarking is first proposed by F. Jordan et al [1] where motion vector of MPEG 4 video stream is slightly modified to embed secret message. Some improved version of the motion vector based watermarking schemes are proposed in [2-5]. These schemes are robust but suffer from lack of payload. Only high value

P. Kalra and S. Peleg (Eds.): ICVGIP 2006, LNCS 4338, pp. 738-748 2006

(C) Springer-Verlag Berlin Heidelberg 2006 
motion vectors can be modified to embed secret message without significant visual distortion. Prediction error based embedding schemes are not very robust as secret message is truncated during the quantization process especially when bit rate is very low. In this paper we propose a new prediction error based adaptive data hiding scheme that can embed relatively large number of bits without significant visual distortion. This blind scheme is relatively robust with respect of the quantization error because embedding is done after quantization. From a pschyco-visual heuristic, the visual distion for injecting slight noise to the block with relatively less inter frame velocity is not very perceptually significant. In proposed scheme, the macro-blocks with less inter frame velocity and with high prediction error are selected for embedding such that embedding distortion may not be visually perceptible. The strength of the error block can be tracked by the quantized DC coefficient of the block. Number of quantized AC coefficients of the prediction error block, which are used for embedding, is adaptively selected on the basis of relative strength of the error block i.e. more numnber of coefficients of the block having higher quantized DC value are used for embedding. Selected number of quantized AC coefficients of prediction error block are modified to embed the secret bits. The experimental result reveals that much more secret bits can be embedded with this proposed scheme than the motion vector based scheme proposed in [5] without significant visual distortion. The visual quality performance comparison is made by compairing PSNR and Watson Metric[6]. Watson Metric which is designed on the basis of characteristics of human visual system, measures the total perceptual error based on frequency sensitivity, luminance sensitivity and contrast masking. The rest of the paper is organized as follows, in section 2 we experimentally shows that more bits can be embedded into error blocks with higher energy (i.e., blocks with larger DC coefficients in transformed domain) without significant perceptual distortion. The details of embedding and extracting schemes are discussed in the section 3. Experimental results will be given in the section 4 and section 5 contains conclusion and future research.

\section{Impact of Embedding in the Prediction Error Block}

The effect of bit embedding in the quantized DCT error blocks are discussed in this section. The main notion of our proposed scheme depends on the following two observations:

- The quantized error blocks with relatively large DC value are more noise tolerant with respect to the Human Visual system (HVS).

- The total perceptual error is directly proportional to the number of secret bits being embedded. So more the bits are embedded, larger is the perceptual error.

In these experimentations, $8 \times 8$ quantization matrix is used for inverse quantization where all matrix elements are sixteen 1 (16). 8x8 IDCT matrix (defined in

${ }^{1}$ As specified for the default quantization table of prediction error used in MPEG 1 standard. 
MATLAB) is used for inverse DCT. The secret bits which are to be embedded are binary random bit stream.

\subsection{Relationship of DC Value and Total Perceptual Error of Prediction Error Block Due to Embedding with Fixed Payload}

In this subsection, we are trying to show that for a fixed embedding change i.e. payload2, the total perceptual error (due to the embedding) of the blocks with relatively higher DC value is lesser than the blocks with relatively lower DC value. To show the above observation experimentally, we collect large number of $8 \times 8$ quantized prediction error blocks of a test video sequence. Now for a fixed embedding change (payload), we calculate the total perceptual error with different DC value for each of the block. The average TPE (total perceptual error) of all the blocks for the different DC value with fixed payload is plotted in figure 1.

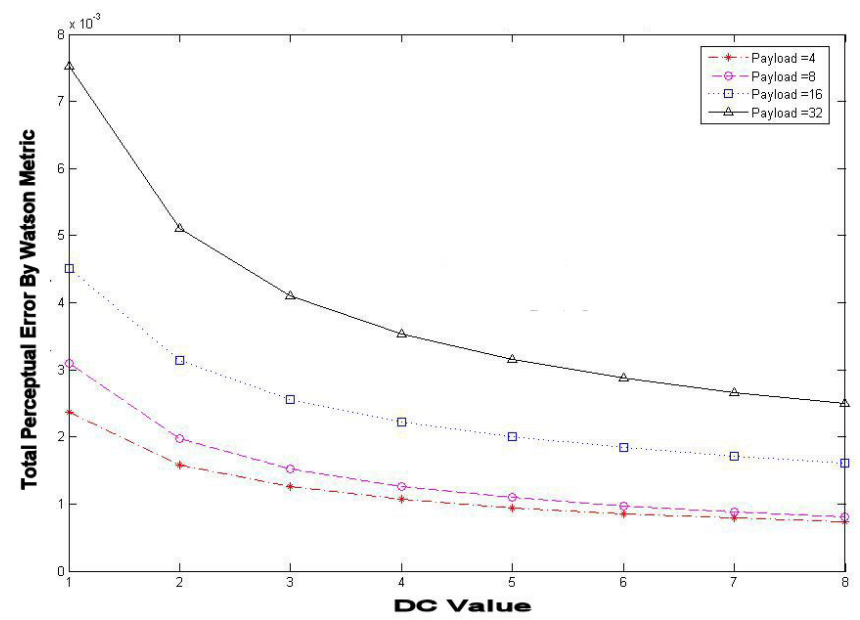

Fig. 1. Relationship between Total Perceptual Error and DC value with fixed Payload

The expermental result shows that the total perceptual error of the block is gradually decreasing with increas of DC value of the block when payload is fixed.

\subsection{Relationship of Payload and Total Perceptual Error of Prediction Error Block Due to Embedding with Fixed DC Value}

In this subsection, we are trying to show that for a fixed DC value, total perceptual error is gradually increasing with increase of embedding change i.e. payload.

${ }^{2}$ Since secret bit stream is random binary sequence, then the payload i.e. number of bits to be embedded is generally doubled of the number of embedding change. 


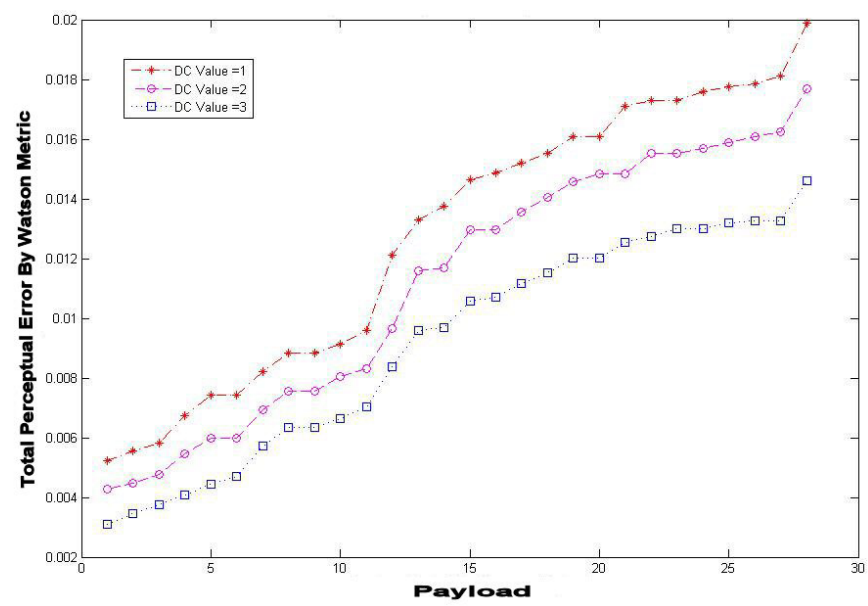

Fig. 2. Relationship between Total Perceptual Error and Payload with fixed DC value

To show this argument experimentally, we collect large number of prediction error blocks of a test video sequence. Now for a fixed DC value, we calculate different total perceptual error of the blocks on different payload. The average total perceptual error of all the blocks for different payload with fixed DC value is plotted in figure 2 .

The experimental result shows that the total perceptual error is gradually increasing with increase of payload when DC value is fixed. From the above observations, payload of a block can be adaptively determined by the equation 1 such that we can control the payload depending on given perceptual error tolerance.

$$
\text { payload }= \begin{cases}0 & \text { if } d c \leq 0 \\ \min \left(\left\lceil\frac{2 \times d c+2}{\lambda}\right\rceil, 32\right) & \text { if } d c>0\end{cases}
$$

where $\lambda$ is a constant and $\lceil\bullet\rceil$ is the Ceiling operator. A small $\lambda$ leads to a large payload so that more data can be embedded with greater total perceptual error to the cover, and vice versa.

\section{Embedding and Extraction Scheme}

\subsection{Embedding Scheme}

In embedding process, the macro-blocks with less inter frame velocity and with high prediction error are selected for embedding such that embedding distortion may not be visually perceptible. The strength of the error block can be tracked by the quantized DC coefficient of the prediction error block. Number of quantized $\mathrm{AC}$ coefficients of the prediction error block, which are used for embedding, is adaptively selected on the basis of relative strength of the error block i.e. more numnber of coefficients, of the block having higher quantized DC value, are 
used for embedding. Selected number of quantized AC coefficients of prediction error block are modified to embed the secret bits. The step by step embedding algorithm is given below:

1. Let the motion vector information of the $i^{\text {th }}$ macro block 3 (B[i]) of a $\mathrm{P}$ frame in a video sequence is

$$
M V[i], 0<i<M B
$$

where $\mathrm{MB}=$ total number of macro blocks presents in the frame.

2. Calculate the magnitude of the motion vector

$$
|M V[i]|=a b s(H[i])+a b s(V[i])
$$

where, $\mathrm{H}[\mathrm{i}]$ is the horizontal component of motion vector in the $i^{\text {th }}$ macroblock; $\mathrm{V}[\mathrm{i}]$ is the vertical component of motion vector in the $i^{\text {th }}$ macroblock of the frame.

3. Given threshold $\epsilon$, select the macroblocks for watermark embedding,

$$
S[i]=F[i] \times B[i]
$$

where $B[i]=i^{t h}$ macro block of the current frame, $S=$ selected macroblocks and $F[i]$ is defined as follows:

$F[i]=\left\{\begin{array}{ll}1 & \text { if }|M V[i]| \leq \epsilon \\ 0 & \text { if }|M V[i]|>\epsilon\end{array} \quad 0<i<M B\right.$

A large $\epsilon$ implies the selection of macro blocks with higher motion vector magnitude for embedding. That leads to greater perceptual distortion.

4. Let $d c(j)$ is the DC coefficient of $j^{\text {th }}$ block among four $8 \times 8$ blocks in the selected macroblock, the number of coefficients $N(j)$ which are modified during embedding is calculated as

$$
N(j)= \begin{cases}0 & \text { if } d c(j) \leq 0 \\ \min \left(\left\lceil\frac{2 \times d c(j)+2}{\lambda}\right\rceil, 32\right) & \text { if } d c(j)>0\end{cases}
$$

where $\lambda$ is a constant and $[\bullet\rceil$ is the Ceiling operator. A small $\lambda$ leads to a large $N(j)$ so that more data can be embedded with greater perceptual error to the cover, and vice versa. Blocks with quantized DC value equal to zero is not used for embedding.

5. Scan the selected error block for $N(j)$ number of quantized AC coefficients in zig zag scan order and embed the current secret bit with the current coefficient in scan order by equation 6. Only quantized AC coefficients are to be modified during embedding. Quantized DC value is kept unchanged. Secret bits are asuumed as random binary digits.

$$
C_{j}^{\prime}(m, n)= \begin{cases}C_{j}(m, n) & \text { if } \bmod \left(\left|C_{j}(m, n)\right|, 2\right)=E \\ C_{j}(m, n)+1 & \text { if } \bmod \left(\left|C_{j}(m, n)\right|, 2\right) \neq E\end{cases}
$$

${ }^{3}$ Size of macro block is $16 \times 16$. 
where $C_{j}(m, n)$ is coefficient positioned in $m^{\text {th }}$ row and $n^{\text {th }}$ coloum of $j^{\text {th }}$ error block where $1 \leq m, n \leq 8 . C_{j}^{\prime}(m, n)$ is corresponding modified coefficient after embedding. $\mathrm{E}$ is the current binary secret bit to be embedded.

\subsection{Extracting Scheme}

Extraction scheme is very simple. The step by step extraction algorithm is given below to extract the hidden bits at the decoder side.

1. The macro blocks are selected at the decoder end by the same equations (3 and 4) as discussed in embedding scheme.

2. The number of modified coefficients can be determined from the unalterd DC value by the same way using equation 5 as in encoder.

3. Scan these coefficients in the same zig zag order as in embedding and determine the secret bits using equation 7 as

$$
E^{\prime}=\operatorname{Mod}\left(C_{j}^{\prime}(m, n), 2\right)
$$

where $C_{j}^{\prime}(m, n)$ is modified coefficients in $j^{\text {th }}$ block in a selected macro block and $E^{\prime}$ is secret bit extracted.

By above extraction method, we can extract all hidden bits without any error.

\section{Expermental Result}

In this section, we will give the experimental results which show that our proposed scheme can embed relatively large number of secret bits without significant visual distortion. In these experimentations we have used 84 frames of suzie, Miss America and salesman test video sequences with fixed bit rate of 1.152

Table 1. Payload of Our Scheme and Scheme proposed in [5]

\begin{tabular}{|c|c|c|c|}
\hline Scheme & suzie & miss america & salesman \\
\hline Our Scheme & 20414 bits & 18714 bits & 20346 bits \\
\hline Scheme in [5] & 3434 bits & 963 bits & 423 bits \\
\hline
\end{tabular}

Mbps, frame rate of 25 frames/sec, PAL video format, 176x144 frame size for testing. The relative payload of our scheme with respect to the scheme proposed in [5] is given in table 1. The comparison plot of PSNR and total perceptual error (by Watson Metric) of Cover 4 , Stegd 5 by our proposed scheme and Stego by scheme proposed in [5] for is given in figure 3. The comparison between our

\footnotetext{
${ }^{4}$ Innocent video frame without any embedded data.

${ }^{5}$ Video frame after embedding secret bits.
} 
scheme and scheme proposed in [5] are given using another 7 video quality metrics[7] such as NMSE(Normalized Mean Square Error), SC(Structural Content), AAD(Absolute Average Difference), CQ(Correlation Quality), NCC(Normalized Cross-Correlation), PMSE (Pulse Mean Square Error)and IF (Image Fidelity). Results shows that our scheme can embed relatively large amount of data maintaining almost same visual quality as proposed in [5] with respect to the above video quality metrics. The comparison plot of NMSE and SC is given in figure 4, comparison plot of $\mathrm{AAD}$ and $\mathrm{CQ}$ is given in figure 5, comparison plot of $\mathrm{NCC}$ and PMSE is given in figure 6 and comparison plot of IF is given in figure 7 .
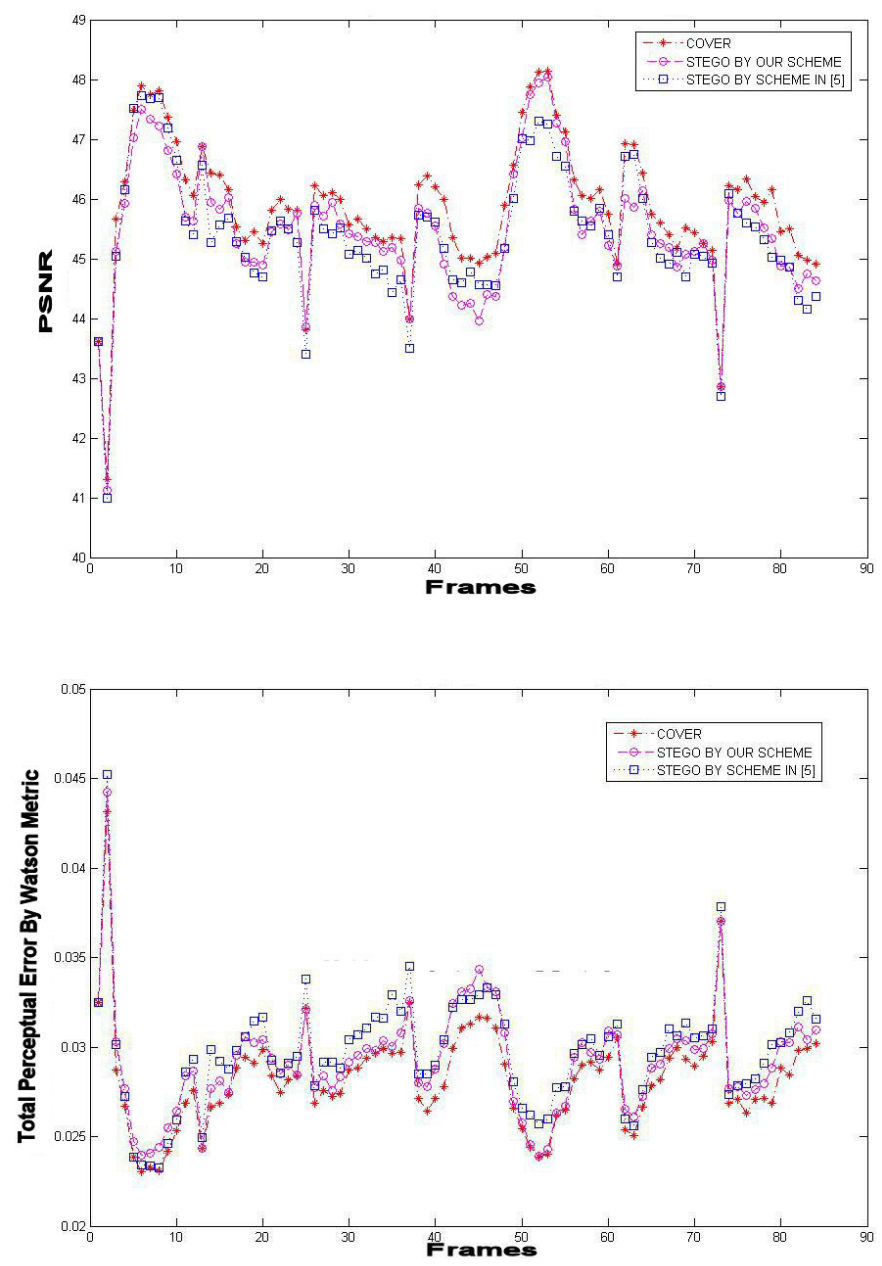

Fig. 3. The PSNR and Total Perceptual Error Plot for Cover, Stego by Our scheme and Stego by schme in [5] 

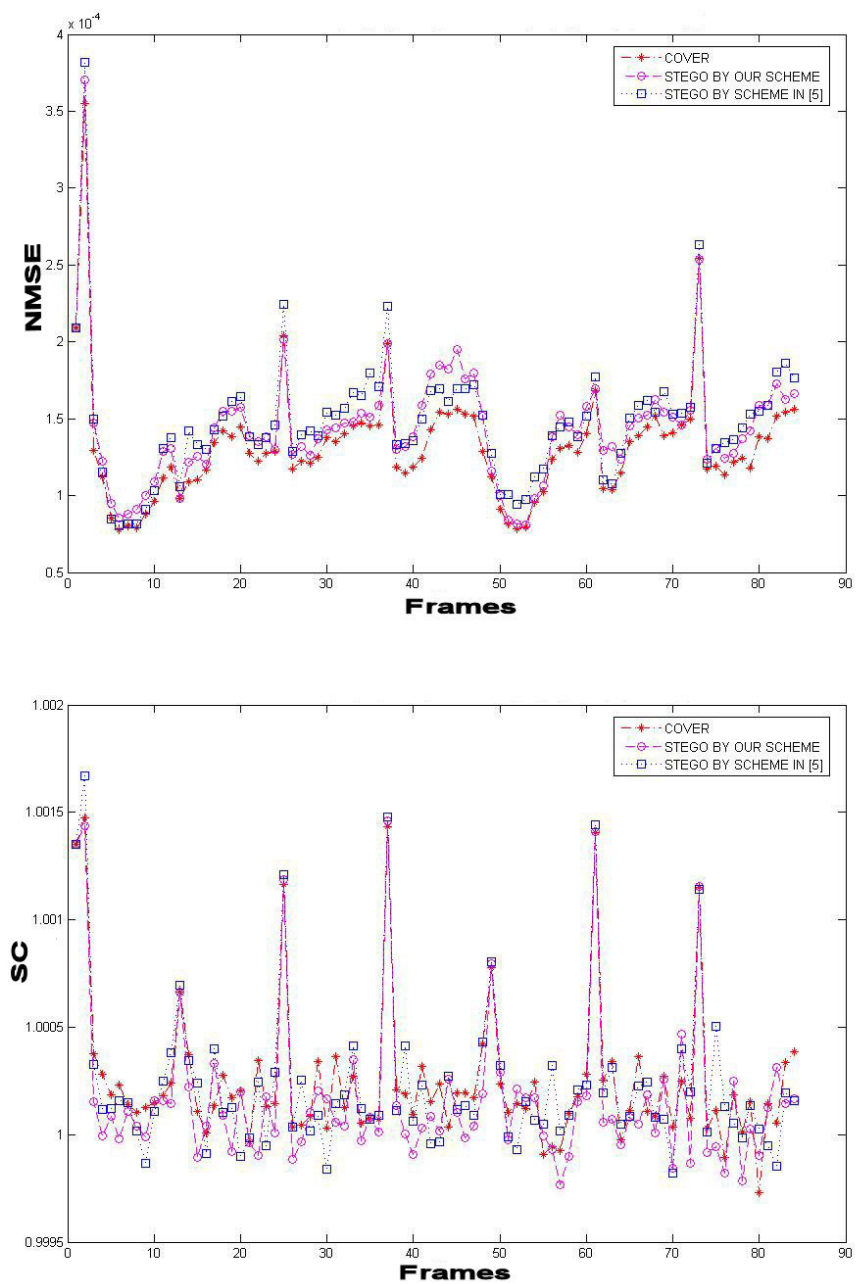

Fig. 4. The Normalized mean square error(NMSE) and Structural Content(SC) Plot for Cover, Stego by Our scheme and Stego by schme in [5]

\section{Conclusion and Future Research}

In this paper we have proposed a new adaptive block based compressed domain data hiding scheme which can embed relatively large number of secret bits without significant perceptual distortion in video domain. Macro blocks are selected for embedding on the basis of low inter frame velocity and high prediction error. The embedding is done by modifying the quantized DCT AC coefficients in the compressed domain. The number of coefficients (both zero and non zero) used in embedding is adaptively determined using relative strength of the 

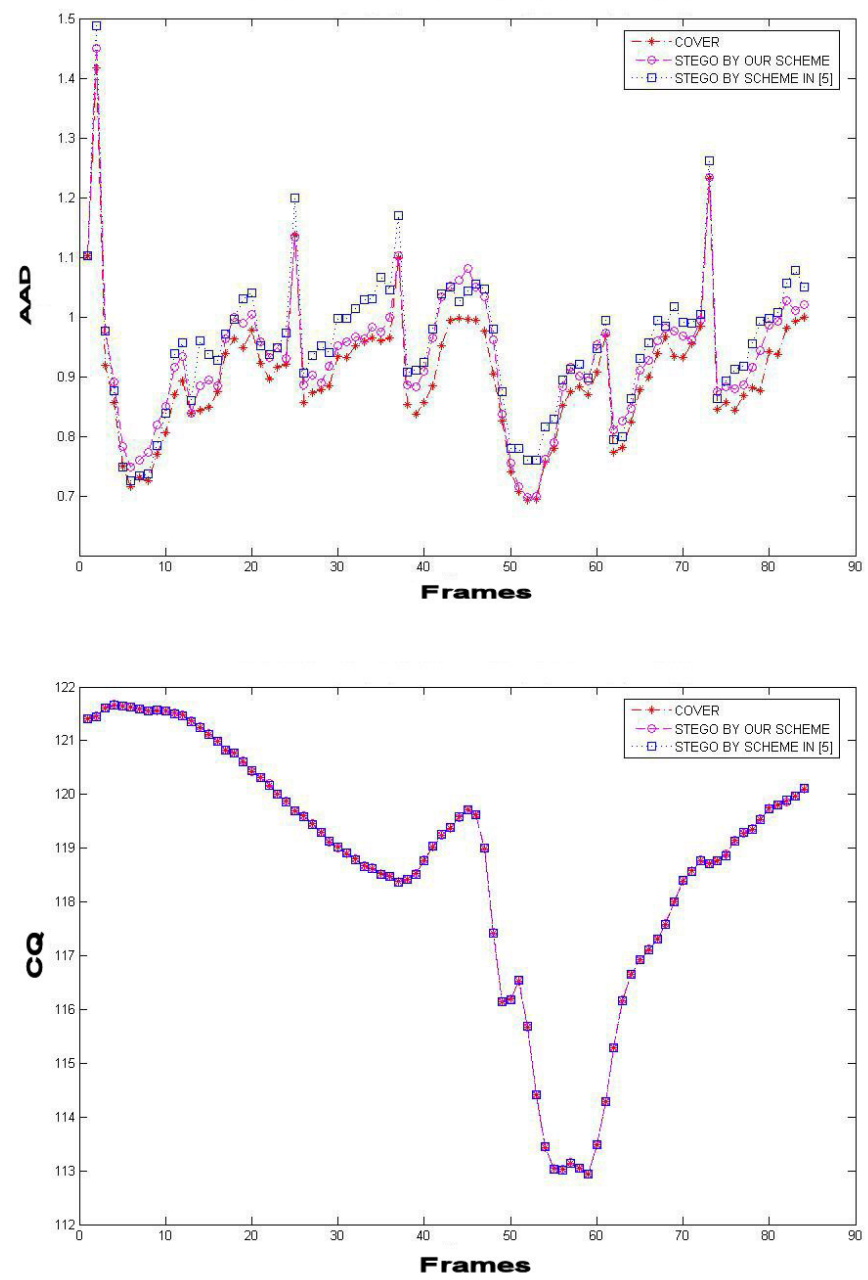

Fig. 5. The Absolute Average Difference(AAD) and Corellation Quality(CQ) Plot for Cover, Stego by Our scheme and Stego by schme in [5]

prediction error block. This blind scheme is relatively more robust with respect of the quantization error because embedding is done after quantization. There may be slightly degradation in visual quality in case of low fixed bit rate video. The proposed scheme is suitable for blind video watermarking where higher payload is necessary and can be used as video steganography as well. In these experimentation, we find that some zone of error block are more noise tolerant so more suitable for embedding. In future, we will concentrate on the problem of adaptive zone selection such that perceptual error due to embedding can be minimized. 

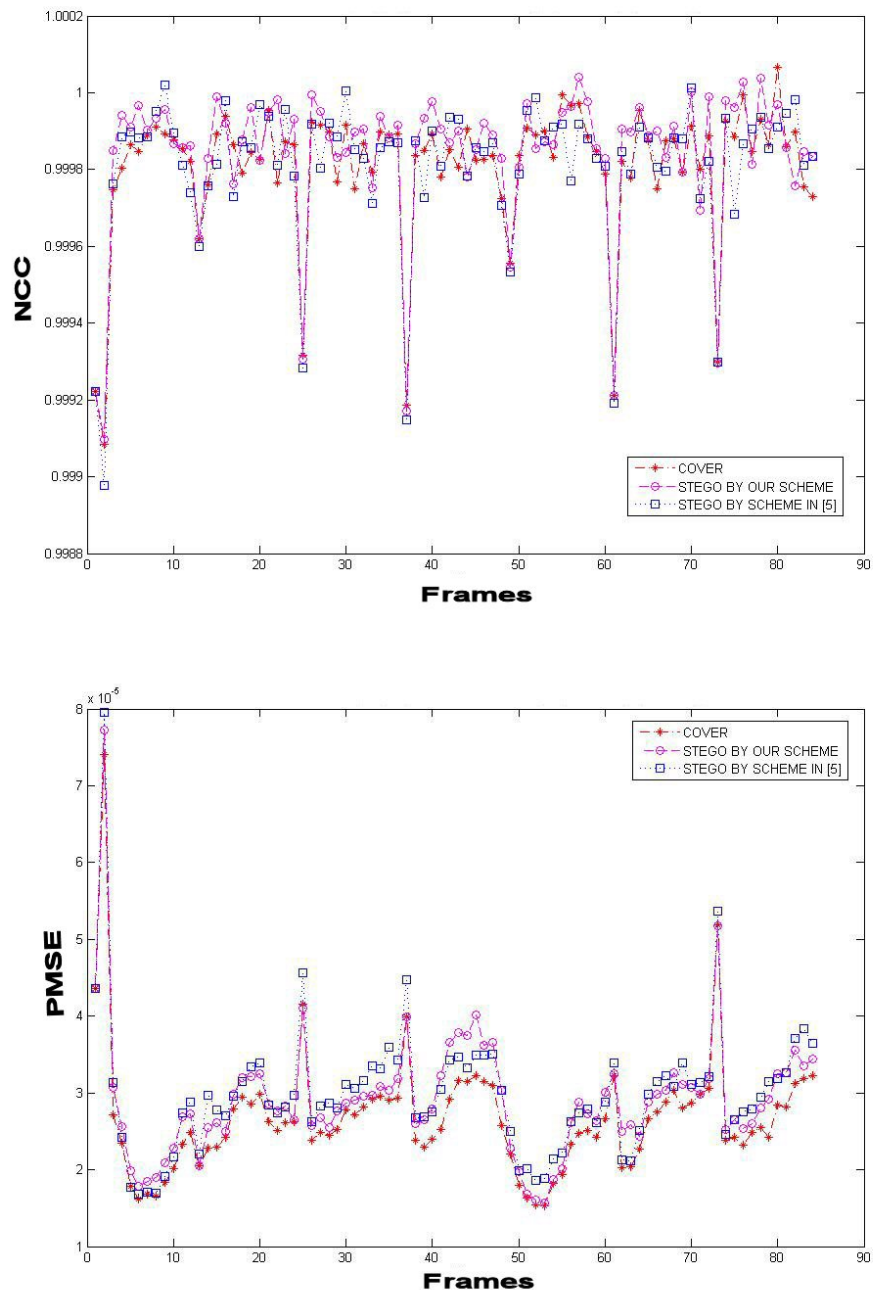

Fig. 6. The Normalized Cross-Corellation(NCC) and Peak Mean Square Error(PMSE) Plot for Cover, Stego by Our scheme and Stego by schme in [5] 


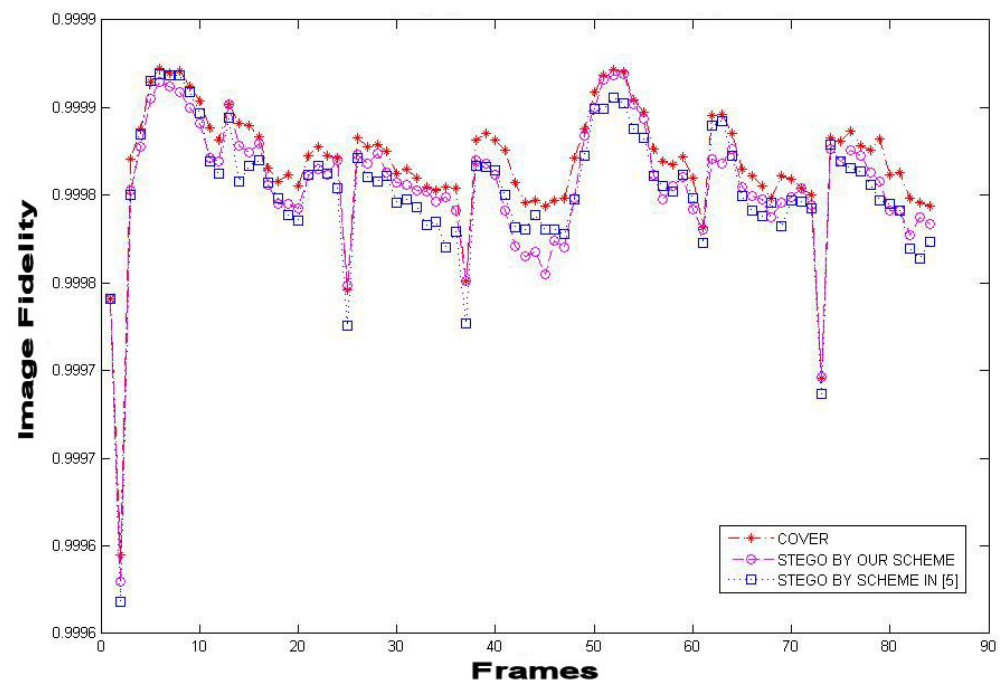

Fig. 7. The Image Fidelity(IF) for Cover, Stego by Our scheme and Stego by schme in $[5]$

\section{References}

1. Kutter M., Jordan F. and Ebrahimi T.: Proposal of a watermarking technique for hiding/retriving data in compressed and decompressed video, Technical reportM2881, ISO/IEC document, JTC1/Sc29/WG11,1997

2. Jun, Z., Jiegu, L., Ling, Z.: Video Watermark technique in motion vector, Proceeding of XIV Brazilian Symposium on Computer graphics and Image Processing, pp. 179$182,2001$.

3. Zhu, Z.,Jiang, G.,Yu, M.,Wu X.: New algorithm for video watermarking, Signal Processing, 2002 6th International Conference on Volume 1, 26-30 Aug. 2002.

4. Liu, L., Liang, H., Niu, X., Yang, Y.: A robust video watermarking in motion vectors, Signal Processing, 2004. Proceedings. ICSP '04. 2004 7th International Conference on Volume 3, 31 Aug.-4 Sept. 2004

5. Zhang, J.,Maitre, H.,Li, J.,Zhang, L.: Embedding watermark in MPEG video sequence, Multimedia Signal Processing, 2001 IEEE Fourth Workshop on 3-5 Oct. 2001

6. Mayache, A., Eude, T., Cherifi, H.: A comparison of image quality models and metrics based on human visual sensitivity, Image Processing, 1998. ICIP 98. Proceedings. 1998 International Conference on 4-7 Oct. 1998.

7. Hazem, M., A., -O.: Evaluation of reconstruction quality in image vector quantisation using existing and new measures, IEE Proc.-Vis. Image Signal Process, Vol. 145, No. 5, October I998 